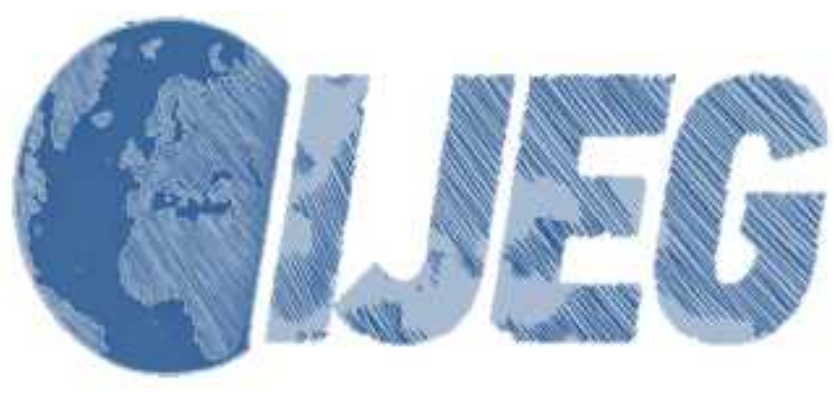

International Journal of Engineering and Geosciences (IJEG),

Vol; 3; , Issue; 1, pp. 036-042, February, 2018, ISSN 2548-0960, Turkey, DOI: $10.26833 /$ ijeg.373152

\title{
AUTOMATIC EXTRACTION OF BUILDING BOUNDARIES FROM HIGH RESOLUTION IMAGES WITH ACTIVE CONTOUR SEGMENTATION
}

\author{
Akbulut, Z., ${ }^{1 *}$ Özdemir, S., ${ }^{1}$ Acar, H., ${ }^{2}$ Dihkan, M., ${ }^{2}$ and Karsli, F., ${ }^{2}$ \\ ${ }^{1}$ Gumushane University, Engineering Faculty, Department of Geomatic Engineering, Gumushane, Turkey \\ (zeynepakbulut/samed.ozdemir @ gumushane.edu.tr); \\ ${ }^{2}$ Karadeniz Technical University, Engineering Faculty, Department of Geomatic Engineering, Trabzon, Turkey \\ (h_acar/mdihkan/fkarsli @ktu.edu.tr)
} ORCID ID 0000-0001-9801-1506; ORCID ID 0000-0001-7217-899X; ORCID ID 0000-0002-2954-7734;
ORCID ID 0000-0002-0027-236X; ORCID ID 0000-0002-0411-3315

*Corresponding Author, Received: 30/12/2017, Accepted: 23/01/2018

ABSTRACT: Building extraction from remotely sensed images plays an important role in many applications such as updating geographical information system, change detection, urban planning, disaster management and 3D building modeling. Automatic extraction of buildings from aerial images is not an easy task because of background complexity, lighting conditions and vegetation cover that reduces separability or visibility of buildings. As a result, automatic building extraction can be a complex process for computer vision and image processing techniques.

In order to overcome this difficulty region-based active contour model was used to automatically detect the boundary of buildings for this study. To extract object boundaries, the model grows or shrinks the initial contour in the image. The main objective of this paper is making active contours algorithm perform without user interaction and to detect automatically initial contours to segment buildings with a software coded in Matlab. This task carried out by morphological operations, band ratio and thresholding methods. In this study, high resolution aerial images with $8 \mathrm{~cm}$ ground sampling distance (GSD) were used. Three separate test zones were selected with varying building level of detail on these images. Finally, it was assessed the accuracy of segmented buildings using Correctness, Completeness and Quality metrics by comparing the results images and manually digitized reference image.

The proposed approach for building extraction from images was shown to be $98 \%$ accurate on buildings with simple geometry and homogeneous roof textures. However accuracy of extracted buildings with heterogeneous roof textures and lighting, and complex geometry is $89 \%$. The results clearly show that automatically calculated initial contour positions work in accordance with the active contour algorithm and easily extraction of the buildings boundaries.

Keywords: Active Contour, Automatic, Building Extraction, High Resolution Image 


\section{INTRODUCTION}

The accurate segmentation of buildings from high resolution aerial images enables many useful applications such as change detection, disaster management, urban planning, updating geographic information system (GIS) databases. According to Wang (2016), extracting of buildings automatically from digital images has been an active research area in both photogrammetry and computer vision communities for decades. However, the automatic extraction of buildings from remotely sensed data is one of the challenging tasks faced by the computer vision and remote sensing communities (Siddiqui, et. al., 2016). This task is challenging due to many reasons such as complexity in the building structures, surrounding environment (highly-dense vegetation, occluded building and hilly scene), poor acquisition of data and registration error between data sources (Siddiqui, et. al., 2016). Also, this is because the remote sensing images are usually characterized by complex data in the form of heterogeneous regions with large intra-class variations and often lower inter-class variations (Alshehhi, et. al., 2017). Thereby automatic building boundary extraction with high accuracy is not a simple task because of background complexity, lighting conditions and vegetation cover that reduces separability or visibility of buildings.

Many researchers have presented methods for the segmentation of buildings from high resolution images. According to Attarzadeh and Momeni (2012), these algorithms have mainly considered radiometric, geometric, edge detection and shadow criteria approaches. Alshehhi et al. (2017) proposed a single patch-based Convolutional Neural Network (CNN) architecture for extraction of roads and buildings from high-resolution remote sensing data. Gilani et al. (2016) developed a methodology to extract and regularize buildings using features from point cloud and ortho-imagery. Wang (2016), developed an approach uses both geometric properties and radiometric properties of building to recognize buildings and delineate their boundaries. Wang's (2016) method consists of automatic generation of high quality point cloud from the imagery, building detection from point cloud, classification of building roof and generation of building outline. Turker and Koc-San (2015), developed an integrated approach for the automatic extraction of rectangular and circular shape buildings from highresolution optical space borne images using the integration of support vector machine (SVM) classification, Hough transformation and perceptual grouping. Song et al. (2015) developed a method to extract buildings using LiDAR data They based their method on distinct topological relationship and characteristics of contour lines and acquired promising results. Ghaffarian (2015) used active contours to segment buildings using automatically extracted initial contour positions based on shadow areas with region growing algorithm. However, the proposed method is incapable of detecting the buildings with no shadows. Kodors et al. (2015) used energy minimization approach to recognize buildings from LiDAR point cloud.

The extraction of 2D images from buildings in a dense urban area is an intricate problem due to the variety of shapes, sizes, colours, and textures (Ghanea et al., 2014). To overcome the problem, they proposed an algorithm which the core components are multispectral binary filtering, sub-clustering and single binary filtering, multiconditional region growing and post-processing.
Awrangjeb et al., (2013), proposed a method for automatic $3 \mathrm{D}$ roof extraction through an effective integration of LiDAR (Light Detection And Ranging) data and multispectral ortho-imagery. Fazan and Poz (2013) used a method utilizing an energy function based on snakes that represents building roof contours in digital images and is optimized with a dynamic programming algorithm. Their results showed that the proposed method satisfactorily performed the task of extracting different building roof contours from digital images. However their method suffered from shadow and another roof nearby. Niveetha and Vidhya (2012) used mathematical morphologic operator to close and eliminate the undesired objects over the building roofs and extracted buildings based on the texture parameters from satellite imagery. Huang and Zuang (2012) proposed a systematic framework for building extraction from high-resolution imagery and aimed to enhance original morphological building index (MBI) algorithm. For this purpose they used a morphological shadow index (MSI) to detect shadows then geometrical index and a vegetation index are used to remove noise from narrow roads and bright vegetation. Ahmadi et al., (2010), proposed a modified active contour model to automatically extract building boundaries on aerial images. All building boundaries are detected by introducing certain points in the buildings' vicinity. They generated the initial curves automatically as a series of regular circles all over the image.

The main objective of this paper is making active contours algorithm perform without user interaction and detect initial contour positions automatically to segment buildings. To detect initial contour positions a series of morphological operations, band ratio and thresholding methods applied to images.

\section{METHODOLOGY}

The proposed approach to automate the active contour algorithm consists of three major steps. These steps are image pre-processing, initial contour creation and building extraction using active contours. Also it was created a Graphical User Interface (GUI) in Matlab software to evaluate all processes in a single frame, to find the optimum initial contour positions and maximum number of iterations, and to obtain the most accurate segmentation results for input image. The flowchart of the methodology for this study is shown in Figure 1 and Figure 2 represents the Matlab interface for the proposed algorithm.

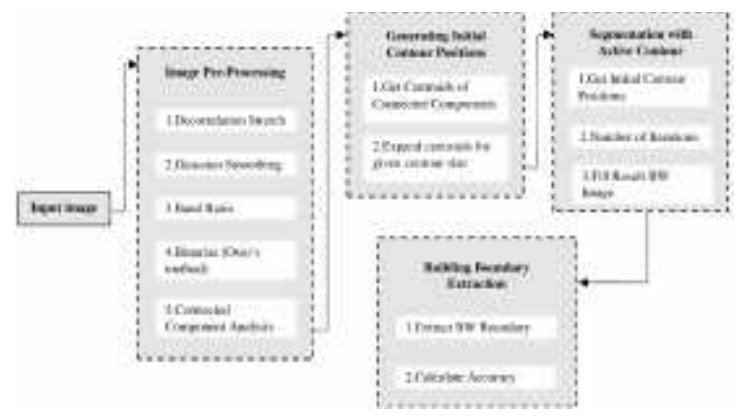

Figure.1 Workflow of automatic building extraction with active contour segmentation 


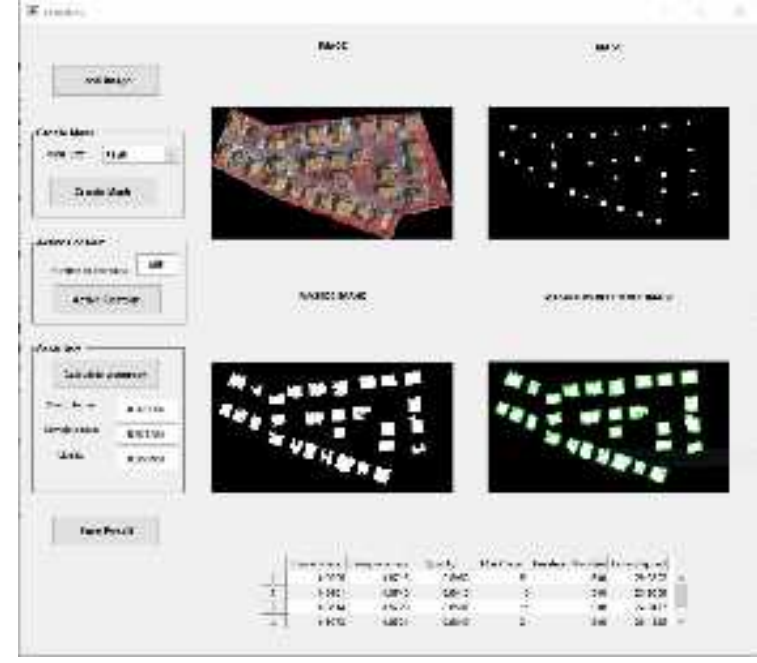

Figure.2 GUI created in Matlab

\subsection{Active Contour Segmentation Algorithm}

Active contours also called snakes was first proposed by Kass et al., (1988). The basic idea in active contour models or snakes is to evolve a curve, subject to constraints from a given image, in order to detect objects in that image. For instance, starting with a curve around the object to be detected, the curve moves toward its interior normal under some constraints from the image, and has to stop on the boundary of the object (Chan and Vese, 2001).

Active contour models can be classified into two categories: parametric snakes and geometrical snakes (Ahmadi et al., 2010). According to Ghaffarian (2015), there are three difficulties with the parametric model. First, the initial contour must, in general, be close to the true boundary, or else it will probably converge to the wrong result. Second, Snake active contours do not progress into boundary concavities. Third, parametric snake active contours are non-convex. Geometrical active contour models consist of two methods: edge based and region based. Region-based geometrical active contours rely on the homogeneity of spatially localized features such as gray level intensity, texture, and other pixel statistics (Ahmadi et al., 2010). Region based active contour models can detect details without significant edges and independent from object boundary waviness. Due to this advantages region based active contour model which also depends on Chan Vese Method is used.

Chan and Vese's (2001) method is a region based geometrical active contour model. The curve's motion is driven by a combination of internal and external forces, which achieve a minimal energy state when the curve/surface reaches the targeted image boundaries (Ahmadi et al., 2010). In Chan and Vese's method they added regularizing terms to the classical active contours to stop the evolving curve on the desired boundary. Energy function for active conlour is shown in Eq. (1).

$$
F_{1}(C)+F_{2}(C)=\int_{\text {inside }(C)}\left|u(x, y)-c_{1}\right|^{2} d x d y
$$

$$
+\int_{\text {outside }(C)}\left|u(x, y)-c_{2}\right|^{2} d x d y
$$

where $\boldsymbol{C}$ is the active contour curve, $\boldsymbol{u}(\boldsymbol{x}, \boldsymbol{y})$ is the pixel values of input image, $c_{1}$ and $c_{2}$ are illustrate the average of pixel vallues inside and outside of $\boldsymbol{C}$, respectively (Chan and Vese, 2001; Almadi et al., 2010).

$$
\begin{aligned}
& F_{1}(C)=\int_{\text {inside(C) }}\left|u(x, y)-c_{1}\right|^{2} d x d y \\
& F_{2}(C)=\int_{\text {outside }(C)}\left|u(x, y)-c_{2}\right|^{2} d x d y
\end{aligned}
$$

Equation (2) is internal energy of curve $\boldsymbol{C}$ and (3) is external energy of curve $\boldsymbol{C}$. Chan and Vese (2001) noted that the boundary of the object $\boldsymbol{C}$ is the minimizer of the fitting energy:

$$
\underset{C}{\left.\ln f_{\left\{F_{1}\right.}(C)+F_{2}(C)\right\} \approx 0 \approx F_{1}(C)+F_{2}(C)}
$$

To explain this if the curve $\boldsymbol{C}$ is outside the object, then $\boldsymbol{F}_{1}(\boldsymbol{C})>0$ and $\boldsymbol{F}_{2}(\boldsymbol{C}) \approx 0$. If the curve $\boldsymbol{C}$ is inside the object, then $\boldsymbol{F}_{1}(\boldsymbol{C}) \approx 0$ but $\boldsymbol{F}_{2}(\boldsymbol{C})>0$. If the curve $\boldsymbol{C}$ is both inside and outside the object, then $\boldsymbol{F}_{1}(\boldsymbol{C})>0$ and $\boldsymbol{F}_{2}(\boldsymbol{C})>0$. Finally, the fitting energy will be minimized if the $\boldsymbol{C}=\boldsymbol{C}$, i.e. if the curve $\boldsymbol{C}$ is on the boundary of the object (Chan and Vese 2001),

In their active contour model Chan and Vese (2001), minimized fitting energy and adided some regularizing terms as showh in Eq. (5);

$$
\begin{aligned}
& \boldsymbol{F}\left(\boldsymbol{C}, c_{1}, c_{2}\right)=\mu \times \text { length }(C)+v \times \text { area }(\text { insideC })+ \\
& \Lambda_{1} \int_{\text {inside }(C)}\left|u(x, y)-c_{1}\right|^{2} d x d y+ \\
& \Lambda_{2} \int_{\text {outside }(c)}\left|u(x, y)-c_{2}\right|^{2} d x d y
\end{aligned}
$$

Regularizing terms include the length of curve $\boldsymbol{C}$ and/or the area inside $\boldsymbol{C}$. In Eq. (5) $\mu, v, \Lambda_{1}$ and $\kappa_{2}$ are fixed parameters and their values are $\mu \geq 0, v \geq 0, \Lambda_{1}>0$ and $\kappa_{2}>0$. Finally, the minimizing problem can be expressed as follows:

$$
{ }_{C}^{i n f} F\left(C, c_{1}, c_{2}\right)
$$

\subsection{Image Pre-processing}

The proposed automatic building extraction approach was tested on high resolution color infrared aerial images. Spatial and radiometric resolution of these images is $8 \mathrm{~cm}$ and 12 bits, respectively. Test images include three different urban residential areas. First area contains 5 buildings with simple geometry and similar roof textures (Figure 3), second area includes 29 buildings with both relatively complex and simple geometry, and also with inhomogeneous roof textures and lighting. The other contains 27 buildings with complex geometry, texture and lighting with respect to second test area. One of the test areas was depicted in Figure 3. 

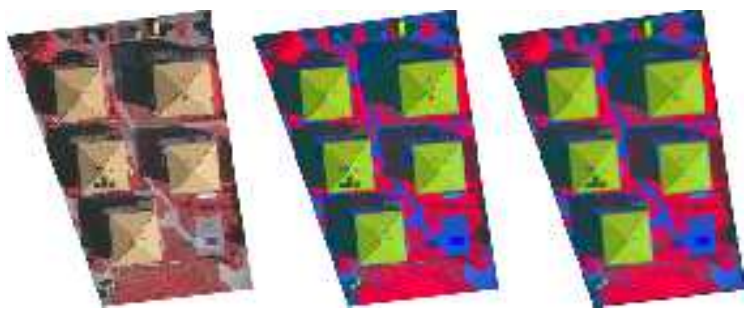

Figure. 3 Test area containing 5 buildings with similar geometric shapes. (a) original image, (b) decorrelation stretched image, (c) Gaussian smoothed image.

Before creating initial contour positions, images were required to be enhanced in order to achieve good results. First, the decorrelation stretch method was used to make the bands of the images more separable. After decorrelation stretching; vegetation, buildings and roads became more distinguished from each other. Images were smoothed to reduce textural details and color transitions. With these enhancements images can be processed with morphological operations more efficiently. After the enhancement operations a band ratio calculated in order to detect approximate building locations. Ratio image then converted to a binary image with Otsu's method. Binarized ratio results contained some undesirable pixel groups that could impair the mask creation process. These pixel groups were removed with a series of morphological operations such as opening and closing.

\subsection{Automatic Creation of Initial Contour Position}

Automatic extraction of buildings based on active contour algorithm was required automatic generation of initial contour positions using centers of points $(x, y)$ which are candidate building pixels. The segmentation process starts at that initial contour positions. To create initial contour positions, firstly, some morphological operations were applied on binary images to separate background from foreground object and so candidate pixels correspondence with buildings were found. Then, pixels were labeled by scanning the imagery pixel by pixel with connected component analysis. After this operation, pixels were grouped by their unique label which is assigned to each class. The centroids $(\mathrm{x}, \mathrm{y})$ were calculated for each approximately detected building pixels group. Finally, all of the centroids were used as initial contour input for active contour segmentation by varying kernel (mask) sizes such as $5 \times 5,11 \times 11,21 \times 21$, and $51 \times 51$ pixels. Optimal kernel size can be selected by users for image segmentation on GUI. Figure 4 shows an example of scene for automatically detected centroids and initial contour positions in a test image.
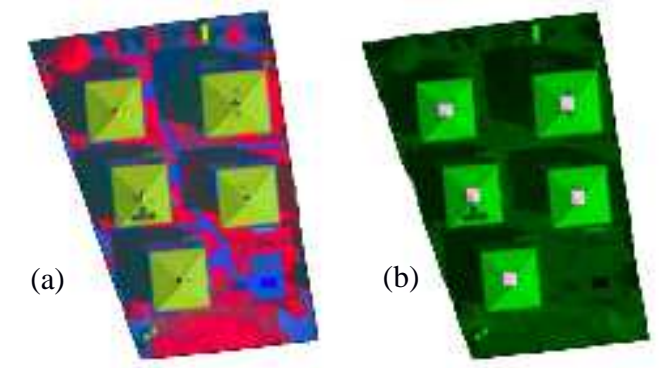

Figure.4 (a) automatically detected centroids (b) initial contour positions

\subsection{Building Extraction with Active Contours}

Active contour algorithm in Matlab requires the input arguments that are image to be segmented, initial contour positions (mask), maximum number of iterations and method. Input image is smoothed image as explain in section 2.2. Second input is initial contour mask mention in section 2.3. Third input is maximum number of iterations to perform in evolution of the segmentation, specified as a numeric scalar (URL-1, 2017). Active contour stops the evolution of the active contour when it reaches the maximum number of iterations (URL-1, 2017). Final input is region based Chan-Vese method that is describe in section 2.1. After preparing initial contours and recognize maximum iteration number image was segmented into two parts as background and foreground.

To accurately recognize boundaries of buildings is depend on well identified mask and maximum iteration number. Active contour also stops the evolution if the contour position in the current iteration is the same as the contour position in one of the most recent five iterations (URL-1, 2017). Optimum mask size and maximum number of iterations for input images were found by trial and error method on graphical user interface. Results images were filled with morphological operations.

Optional parameters for active contour algorithm in Matlab software are Smooth Factor and Contraction Bias. Smooth Factor is degree of smoothness or regularity of the boundaries of the segmented regions (URL-1, 2017). Contraction Bias is tendency of the contour to grow outwards or shrink inwards and this parameter does not guarantee that the contour contracts (or expands) (URL-1, 2017). For this reason, the default values of Contraction Bias and Smooth Factor were used in applying Chan-Vese method. Finally, output segmented binary image was obtained.

\section{RESULTS AND DISCUSSION}

The accuracy assessment of proposed approach were carried out by comparing automatically extracted buildings with manually digitized reference images using Completeness, conectness and Quality metrics as shown in Eq (7), (8) and (9).

$$
\begin{aligned}
& \text { Completeness }=\frac{\|T P\|}{\|T P\|_{i}\|F \mathrm{FN}\|} \\
& \text { Correctness }=-\frac{\|T P\|}{\|T P\|+\|F P\|}
\end{aligned}
$$




$$
\text { Quality }=\frac{\left\|T{ }^{\mu}\right\|}{\|T F\|+|| F N\|+\| F F \|}
$$

In Eqs. (7), (8), and (9), an entity classified as an object that also corresponds to an object in the reference is classified as a true positive (TP). A false negative (FN) is an entity corresponding to an object in the reference that is classified as background, and a false positive (FP) is an entity classified as an object that does not correspond to an object in the reference. A true negative (TN) is an entity belonging to the background both in the classification and in the reference data (Rutzinger et al. 2009; Shufelt 1999; Karsli et al. 2016).

The results of automatically extracted building boundaries were shown in Figs. (5), (6) and (7). Quantitative results for each test area were depicted in Table (1), (2) and (3).

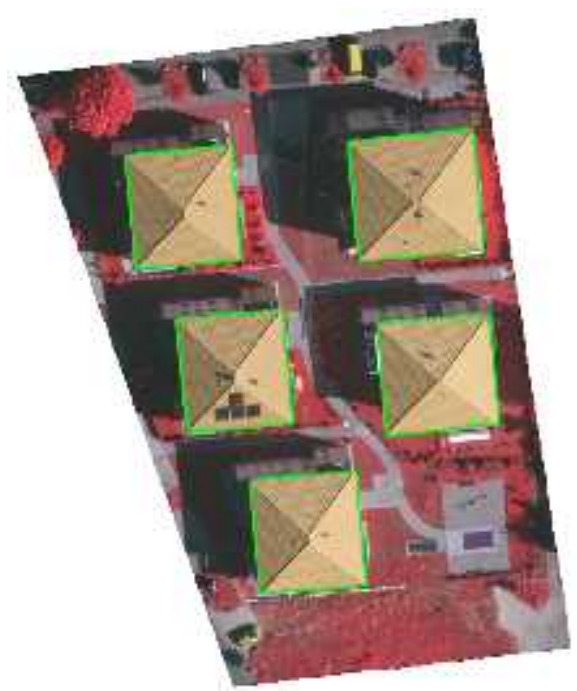

Figure.5 Results of first test area

Table 1 Accuracy assessment of first region with 5 buildings

\begin{tabular}{ccccc}
\hline Correctness & Completeness & Quality & $\begin{array}{c}\text { Mask } \\
\text { size }\end{array}$ & Iteration \\
\hline 0,872 & 0,995 & 0,868 & $5 \times 5$ & 200 \\
0,889 & 0,994 & 0,884 & $11 \times 11$ & 200 \\
0,918 & 0,993 & 0,912 & $21 \times 21$ & 200 \\
0,981 & 0,990 & 0,971 & $51 \times 51$ & 200 \\
0,970 & 0,990 & 0,960 & $5 \times 5$ & 250 \\
0,975 & 0,990 & 0,966 & $11 \times 11$ & 250 \\
0,984 & 0,989 & 0,973 & $21 \times 21$ & 250 \\
0,989 & 0,987 & 0,977 & $51 \times 51$ & 250 \\
0,989 & 0,987 & 0,977 & $5 \times 5$ & 300 \\
\hline
\end{tabular}

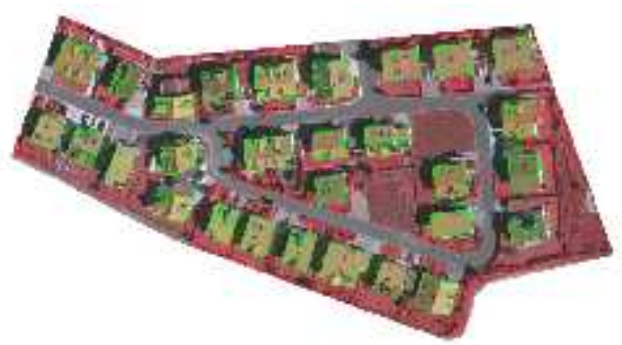

Figure.6 Results of second test area

Table 2 Accuracy assessment of second region with 29 buildings

\begin{tabular}{|c|c|c|c|c|}
\hline Correctness & Completeness & Quality & $\begin{array}{c}\text { Mask } \\
\text { size }\end{array}$ & Iteration \\
\hline 0.818 & 0.974 & 0.801 & $5 \times 5$ & 500 \\
\hline 0.871 & 0.973 & 0.851 & $11 \times 11$ & 500 \\
\hline 0.907 & 0.972 & 0.885 & $21 \times 21$ & 500 \\
\hline 0.921 & 0.971 & 0.896 & $51 \times 51$ & 500 \\
\hline 0.922 & 0.971 & 0.897 & $51 \times 51$ & 750 \\
\hline 0.922 & 0.971 & 0.898 & $51 \times 51$ & 1000 \\
\hline
\end{tabular}

Figure.7 Results of third test area

Table 3: Accuracy assessment of third region with 27 buildings

\begin{tabular}{ccccc}
\hline Correctness & Completeness & Quality & $\begin{array}{c}\text { Mask } \\
\text { size }\end{array}$ & Iteration \\
\hline 0.871 & 0.966 & 0.845 & $5 \times 5$ & 500 \\
0.883 & 0.965 & 0.856 & $11 \times 11$ & 500 \\
0.892 & 0.965 & 0.864 & $21 \times 21$ & 500 \\
0.915 & 0.963 & 0.884 & $51 \times 51$ & 500 \\
0.923 & 0.961 & 0.890 & $11 \times 11$ & 750 \\
0.923 & 0.961 & 0.890 & $51 \times 51$ & 750 \\
0.923 & 0.961 & 0.890 & $21 \times 21$ & 1000 \\
0.923 & 0.961 & 0.890 & $11 \times 11$ & 1000 \\
0.923 & 0.961 & 0.890 & $51 \times 51$ & 1000 \\
\hline
\end{tabular}


According to the results, optimum parameter values were determined with various combinations of maximum number of iterations and initial contour size for each test area to increase the accuracy of automatic building extraction. The trials were terminated when there is no improvement over optimum values. For instance, after performing active contour segmentation with $11 \times 11$ initial contour size and 750 iterations, results compared to other segmentations which carried out with greater values for iteration number and initial contour size showed no significant improvement as shown in Table 3. Moreover in Table 1 , same results were acquired both $51 \times 51$ initial contour size with 250 iterations and $5 \times 5$ initial contour size with 300 iterations. As a result, selecting small initial contour size with a suitable iteration number can give the same results with a bigger initial contour size and lower iteration number.

To get desired segmentation results, there is no need to initial contour positions be close the object boundaries. Therefore, an initial contour selected at the center of the object to be segmented can be sufficient. Thus, high segmentation accuracy can be acquired with small initial contour created near the object center and a greater number of iteration. Accuracy for each test area was shown in Table 4.

Table 4 The best results for each test area

\begin{tabular}{cccc}
\hline Test Areas & Correctness & Completeness & Quality \\
\hline First & 0,989 & 0,987 & 0,977 \\
\hline Second & 0.922 & 0.971 & 0.898 \\
\hline Third & 0.923 & 0.961 & 0.890 \\
\hline
\end{tabular}

While spectrally inhomogeneous and geometrically complex buildings were extracted with lower accuracy (Fig. 8 and 9), but buildings with spectrally homogeneous roof were extracted with higher accuracy (fig. 10). The reason for low extraction accuracy depends on the objects like solar panels or windows locating near the roof edges and in such situation active contour algorithm.
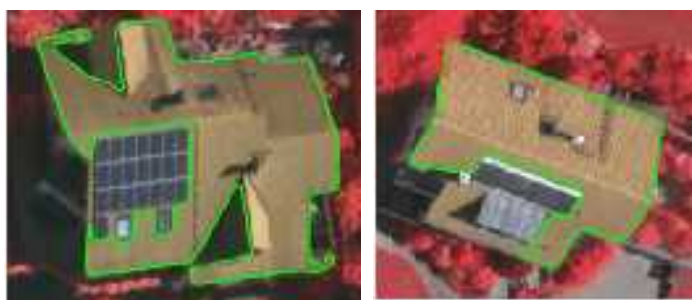

Figure. 8 Objects on roofs such as sun panels affect the accuracy of active contours
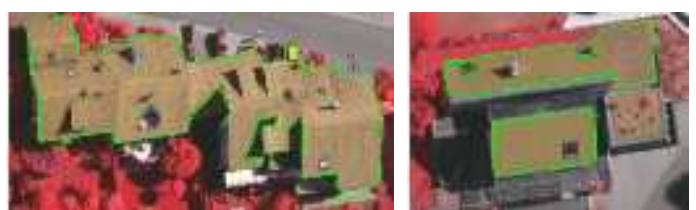

Figure.9 Shadowed zones on roofs especially near the edge also impair active contours, spectrally indistinctive pixels mixed with roof pixels
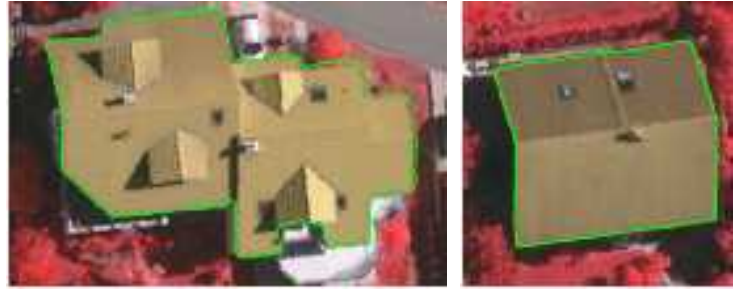

Figure.10 Decently illuminated roofs without prominent shadow areas can be accurately extracted by active contours, simple roof shapes can be extracted easily

\section{CONCLUSIONS AND FUTURE WORKS}

This study presents to create automatic initial contour positions for the extraction of buildings from high resolution aerial images instead of manually defining the initial contour positions in region based active contour algorithm. To do so, first the building candidate pixels were determined with morphological operations and labeled as the regions of building from binary images. Then, each building candidate pixel group's centroids were calculated. These centroids were used as initial contour positions by upsizing their dimensions with predetermined values such as $5 \times 5,11 \times 11,21 \times 21$, and $51 \times 51$ to run active contour model. To obtain desirable segmentation results, trial and error approach were implemented to find optimum initial contour size and iteration number in GUI.

With the proposed approach, all of the buildings were extracted from images. However, shadow effects and mixed pixels couldn't be avoided due to spectral characteristics of the environment such as roads and vegetation. As a result of previously mentioned issues, some of the detected buildings have jagged boundaries because of shadow effects and some of the building boundaries expanded to non-building areas with spectrally similar characteristics. Also, if the initial curve overlaps with a chimney, window or a solar panel then the curve created by active contour algorithm retracts inward and disappear. To overcome this problem, initial curve size must be large enough to surround such objects. Nevertheless for homogeneous surfaced building roofs, overall segmentation quality does not affected by the size of initial contour.

Because of the fact that the main contribution in this study is to automatically create initial contour positions, it was not taken into consideration of the shadow regions and smoothness of the boundaries. Thus, as a future work we are going to smooth jagged boundaries and remove shadows effects on building roofs. Also, it is consider that Digital Elevation Model (DEM) covering the images will be included into dataset as a one layer to extract building boundaries with different roof textures and colors with active contour algorithm. 


\section{REFERENCES}

Ahmadi, S., Zoej, M. J. V., Ebadi, H., Moghaddam, H. A., Mohammadzadeh, A., 2010, Automatic urban building boundary extraction from high resolution aerial images using an innovative model of active contours, International Journal of Applied Earth Observation and Geoinformation 12 (2010) 150-157.

Alshehhi, R., Marpu, P. R., Woon, W. L., Mura, M. D., 2017, Simultaneous extraction of roads and buildings in remote sensing imagery with convolutional neural networks, ISPRS Journal of Photogrammetry and Remote Sensing, 130 (2017) 139-149.

Attarzadeh, R., Momeni, M., 2012, Object-Based Building Extraction from High Resolution Satellite Imagery, International Archives of the Photogrammetry, Remote Sensing and Spatial Information Sciences, Volume XXXIX-B4, 2012 XXII ISPRS Congress, 25 August - 01 September 2012, Melbourne, Australia.

Awrangjeb, M., Fraser, C. S., Lua, G., 2013, Integration of Lidar Data And Orthoimage For Automatic 3D Building Roof Plane Extraction, Multimedia and Expo (ICME), 2013 IEEE International Conference on, doi: 10.1109/ICME.2013.6607612.

Chan, T. F. and Vese, L. A., 2001, Active contours without edges, IEEE Transactions on Image Processing, Volume 10, Issue 2, pp. 266-277.

Fazan, A. J., Poz, A. P. D., 2013, Rectilinear building roof contour extraction based on snakes and dynamic programming, International Journal of Applied Earth Observation and Geoinformation, doi: 10.1016/j.jag.2013.03.003.

Ghaffarian, S., 2015, An Approach For Automatic Building Extraction From High Resolution Satellite Images Using Shadow Analysis And Active Contours Model, Master Thesis, Hacettepe University, Ankara, Turkey.

Ghanea, M., Moallem P., Momeni, M., 2014, Automatic Building Extraction in Dense Urban Areas through GeoEye Multi-Spectral Imagery, International Journal of Remote Sensing 35 (13): 5094-5119. doi:10.1080/01431161.2014.933278

Gilani, S. A. N., Awrangjeb, M., Guojun, L., 2016, An Automatic Building Extraction and Regularisation Technique Using LiDAR Point Cloud Data and Orthoimage, Remote Sensing 2016, 8(3), 258, doi: $10.3390 / \mathrm{rs} 8030258$.

Huang, X., Zhang, L., 2012, Morphological Building/Shadow Index for Building Extraction From High-Resolution Imagery Over Urban Areas, IEEE Journal of Selected Topics in Applied Earth Observations and Remote Sensing, Volume: 5, Issue: 1, Feb. 2012, doi: 10.1109/JSTARS.2011.2168195.
Karsli, F., Dihkan, M., Acar, H., Ozturk, A., 2016, Automatic building extraction from very high-resolution image and LiDAR data with SVM algorithm, Arabian Journal of Geosciences, doi: 10.1007/s12517-016-2664-7.

Kass, M., Witkin, A., Terzopoulos, D., 1988, Snakes: Active contour models, International Journal of Computer Vision, 321-331.

Kodors, S., Ratkevics, A., Rausis, A., Buls J., 2015, Building Recognition Using LiDAR and Energy Minimization Approach, Procedia Computer Science, Volume 43, 2015, Pages 109-117.

Niveetha, M. A., Vidhya, R., 2012, Automatic Building Extraction Using Advanced Morphological Operations and Texture Enhancing, Procedia Engineering 38:35733578 .

Rutzinger, M, Rottensteiner, F, Pfeifer, N., 2009, A comparison of evaluation techniques for building extraction from airborne laser scanning, IEEE Journal of Selected Topics in Applied Earth Observations and Remote Sensing 2(1):11-20.

Siddiqui, F. U., Teng, S. W., Awrangjeb, M., Lu G., 2016, A Robust Gradient Based Method for Building Extraction from LiDAR and Photogrammetric Imagery, Sensors 2016, 16(7), 1110; doi:10.3390/s16071110.

Song, J., Wu, J., Jiang, Y., 2015, Extraction and reconstruction of curved surface buildings by contour clustering using airborne LiDAR data, Optik 126 (2015), 513-521.

Shufelt, J. A., 1999, Performance evaluation and analysis of monocular building extraction from aerial imagery. Pattern Analysis and Machine Intelligence, IEEE Transactions on 21(4):311-326.

Turker, M., Koc-San, D., 2015, Building extraction from high-resolution optical spaceborne images using the integration of support vector machine (SVM) classification, Hough transformation and perceptual grouping, International Journal of Applied Earth Observation and Geoinformation, 34 (2015) 58-69.

URL-1, 2017, https://www.mathworks.com/help/images/re f/activecontour.html, [12 November 2017].

Wang, Y., 2016, Automatic Extraction of Building Outline From High Resolution Aerial Imagery, The International Archives of the Photogrammetry, Remote Sensing and Spatial Information Sciences, Volume XLI-B3, 2016 XXIII ISPRS Congress, 12-19 July 2016, Prague, Czech Republic.

Copyright (C) International Journal of Engineering and Geosciences (IJEG). All rights reserved, including the making of copies unless permission is obtained from the copyright proprietors. 